\title{
Modeling of Traffic Excitation for System Identification of Bridge Structures
}

\author{
Yangbo Chen, Maria Q. Feng* \\ Department of Civil and Environmental Engineering, UC Irvine, Irvine, CA 92697-2175, USA
}

$\&$

Chin-An Tan

Department of Mechanical Engineering, Wayne State University, Detroit, MI 48202, USA

\begin{abstract}
In long-term health monitoring of bridge structures, system identification is often performed based only on the system output (bridge vibration responses) because the system input (traffic excitation) is difficult to measure. To facilitate the identification of the bridge properties, traffic excitation is commonly modeled as spatially uncorrelated white noise. A physical model of a stationary stream of vehicles (moving loads) arriving in accordance with a Poisson process, traversing an elastic beam, shows that the traffic excitation is spatially correlated. Employing the dynamic nodal loading approach, this spatial correlation results in a frequency-dependent excitation spectrum density matrix, and shifts the response spectra obtained from those excited by spatially uncorrelated white noise. It is shown that the application of system identification techniques based on the conventional excitation model may result in misleading structural properties. Hence, this study further proposes an output-only gray-box identification technique for bridge structures, in which knowledge about the nature of the traffic excitation, such as its spatial correlation, is implanted into an autoregressive-movingaverage (ARMA) model. The identifiability of the ARMA model so constructed is assured and the feasibility of the proposed identification technique is demonstrated by a numerical example.
\end{abstract}

\footnotetext{
*To whom correspondence should be addressed. E-mail: mfeng@ uci.edu.
}

\section{INTRODUCTION}

Continuous or periodic measurements of the vibration of highway bridges under normal traffic excitations are desirable for the long-term monitoring of structural performance. Such measurements can readily be conducted on instrumented bridges, without interrupting the traffic, to provide valuable information on the in situ dynamic behavior of the bridges in normal service. A key difficulty in the measurement process of bridge vibration remains, however, in that the excitation loads are neither controllable nor (easily) measurable. Thus, to extract the structural properties of the bridge from the measurements, system identification is performed based only on the measured time histories of the bridge response (system output), without measurements of the traffic excitations (system input). As a result, to facilitate such output-only identification of structural properties, estimation models and/or assumptions on the input characteristics must be established based on some a priori knowledge of the excitations because bridge responses depend not only on the dynamic properties of the bridge structure but also on the excitations.

In recent years, several output-only identification techniques have been developed. These include the natural excitation technique (James et al., 1996; Shen et al., 2003; Caicedo et al., 2004), the frequency domain decomposition (FDD) (Brincker et al., 2001; Feng et al., 
2002), the subspace decomposition (Peeters et al., 2001), the random decrement technique (Asmussen and Brincker, 1996; Feng and Kim, 1998), and various types of autoregressive-moving-average (ARMA) model fitting techniques (Garibaldi et al., 1998; Jensen et al., 1992; Huang, 2001). A common assumption of these techniques is the spatially uncorrelated white noise input model (referred to hereafter as the conventional excitation model), which mathematically simplifies the identification. This article evaluates the applicability of this assumption in the context of bridge structural health monitoring.

We formulate the problem by first modeling the traffic excitation as a stationary stream of vehicles, arriving in accordance with a Poisson process, traversing an elastic beam. With this formulation, the traffic excitation on a bridge is found to be spatially correlated. This is expected because the same vehicles will pass through all longitudinal positions on the bridge, and traffic-induced excitation at one position can thus be viewed as a delayed process of the excitations at preceding positions. This spatial correlation results in an excitation spectrum density matrix (SDM) whose off-diagonal entries are nonzero functions of the frequency. Numerical results show that direct application of output-only identification techniques based on the conventional excitation model to responses of bridges subjected to spatially correlated excitations can result in misleading conclusions on the dynamic properties of the bridge structure.

A physics-based traffic excitation model is proposed incorporating the spatial correlated nature. Employing this model, an output-only gray-box system identification scheme is proposed and its feasibility is demonstrated in this article. The structural response conforms to an ARMA model. The MA part is parameterized by embedding some a priori knowledge on the excitation, such as its spatial correlation, and the AR part is parameterized by our knowledge about the structural properties. This parameterization assures the identifiability of the ARMA model such that a unique identification of the structural properties and excitation characteristics can be achieved. A numerical example of a bridge structure subjected to a spatially correlated excitation is presented to demonstrate the feasibility and efficacy of the proposed identification technique.

\section{PROPOSED PHYSICS-BASED TRAFFIC EXCITATION MODEL}

\subsection{Problem formulation: Beam moving-force model}

When a vehicle traverses a bridge, both the bridge and the vehicle will vibrate. It has been shown that, for shortto mid-span highway bridges, in the normal range of

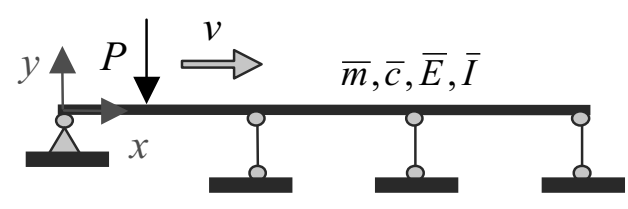

Fig. 1. Beam moving-force model.

vehicle speeds, large dynamic tire forces are due primarily to road surface irregularity rather than bridge-vehicle interactions (Yang et al., 2000; Pesterev et al., 2003; Schenk and Bergman, 2003; Pesterev et al., 2004). As a result, decoupling the bridge-vehicle system leads to the beam moving-force model, that is, the bridge (modeled as an elastic beam) is subjected to a time-variant tire force $P(t)$ moving across it (Cebon, 1999; Pan and $\mathrm{Li}$, 2002). In this article, $P(t)$ is taken as a constant $P$ during the derivation, which can account for the static tire force $M_{v} g$, where $M_{v}$ is the mass of the vehicle. The model is schematically shown in Figure 1 , where $\bar{m}, \bar{c}, \bar{E}$, and $\bar{I}$ are the mass per unit length, the damping coefficient, the Young's modulus, and the cross-sectional moment of inertia of the bridge, respectively, $v$ is the speed of the vehicle and $y_{b}$ is the vertical deflection of the bridge with respect to the equilibrium position.

The governing equation of motion for $y_{b}(t)$ is

$$
\begin{aligned}
\bar{m} & \frac{\partial^{2}}{\partial t^{2}} y_{b}(x, t)+\bar{c} \frac{\partial}{\partial t} y_{b}(x, t) \\
& +\overline{E I} \frac{\partial^{4}}{\partial x^{4}} y_{b}(x, t)=-\delta(x-v t) P
\end{aligned}
$$

When multiple vehicles move across the bridge, the vehicle arrival can be assumed to follow a Poisson process with mean rate $\lambda$ (Shinozuka and Kobori, 1972; Turner and Pretlove, 1988). Therefore, the right hand side of Equation (1) can be replaced by

$$
F_{x}(t)=-\sum_{i=1}^{N(t)} P_{i} \delta\left[x-v\left(t-\tau_{i}\right)\right]
$$

where $F_{x}(t)$ is the time history of traffic excitation at location $x$ on the bridge; $N(t)$ is the number of vehicle arrivals during time interval $[0, t)$, and $\tau_{1}, \tau_{2}, \ldots, \tau_{i}, \ldots, \tau_{N(t)}$ are the sequence of arrival times. $P_{i}$ is the static tire force of the $i$ th vehicle. $\left\{P_{i}\right\} \quad(i=1$ to $N(t)$ ) are independent random variables identically distributed as a random variable $P$, independent to the Poisson process. Equation (2) defines a filtered Poisson process $F_{x}(t)$ (Parzen, 1962). On the basis of Campbell's theorem (Parzen, 1962) for filtered Poisson processes, the statistical characteristics of $F_{x}(t)$, including its mean, variance, and covariance at different locations of $x$, can be calculated. Hence, the SDM for the traffic excitation on the bridge can be derived. 


\subsection{Construction of physics-based traffic excitation SDM}

Structural analyses of multispan bridges and other complex structures are usually conducted by finite element method (FEM). To utilize most of the existing finite element programs for computing the bridge response to moving vehicles, Pan and $\mathrm{Li}$ (2002) proposed the dynamic nodal loading (DNL) method. In the DNL method, the time-variant moving force is converted into load histories at each of the nodes in the finite element model based on the equivalent nodal forces (ENFs) concept. In this way, the moving load problem is transformed into a time-history analysis problem, which can easily be incorporated into most available FEM codes, yielding results with good engineering precision (Pan and $\mathrm{Li}, 2002$ ). In this article, the DNL method is implemented in the procedure of constructing the SDM of excitation due to moving vehicles.

Let the bridge girders be modeled by beam elements with two degrees of freedom at each node, that is, the vertical displacement $y_{i}$ and the in-plane rotation $\theta_{i}$ at node $i$. It has been established that a vertical force $P$ applied within the beam element is equivalent to the combined action of a nodal shear $Q_{i}$ and a nodal moment $M_{i}$ (the ENFs) acting at the nodes (Hibbeler, 2002). When the force moves across the beam elements, the ENFs are functions of the position of the moving force.

When the moving force $P$ is a unit constant, the ENFs are

$Q_{i}(t)=W_{i}^{Q}(t)$

$=\left\{\begin{array}{cc}0 & \left(t \leq \frac{x_{i}-l_{i-1}}{v}\right) \\ \frac{\left(l_{i-1}-x_{i}+v t\right)^{2}}{l_{i-1}^{2}}\left[1+\frac{2\left(x_{i}-v t\right)}{l_{i-1}}\right] & \left(\frac{x_{i}-l_{i-1}}{v}<t \leq \frac{x_{i}}{v}\right) \\ \frac{\left(l_{i}+x_{i}-v t\right)^{2}}{l_{i}^{2}}\left[1+\frac{2\left(v t-x_{i}\right)}{l_{i}}\right] & \left(\frac{x_{i}}{v}<t \leq \frac{x_{i}+l_{i}}{v}\right) \\ 0 & \left(\frac{x_{i}+l_{i}}{v}<t\right)\end{array}\right.$

$$
\begin{aligned}
& M_{i}(t)=W_{i}^{M}(t) \\
& =\left\{\begin{array}{cc}
0 & \left(t \leq \frac{x_{i}-l_{i-1}}{v}\right) \\
-\frac{\left(l_{i-1}-x_{i}+v t\right)^{2}}{l_{i-1}^{2}}\left(x_{i}-v t\right) & \left(\frac{x_{i}-l_{i-1}}{v}<t \leq \frac{x_{i}}{v}\right) \\
\frac{\left(l_{i}+x_{i}-v t\right)^{2}}{l_{i}^{2}}\left(v t-x_{i}\right) & \left(\frac{x_{i}}{v}<t \leq \frac{x_{i}+l_{i}}{v}\right) \\
0 & \left(\frac{x_{i}+l_{i}}{v}<t\right)
\end{array}\right.
\end{aligned}
$$

where $v, l_{i}$, and $l_{i-1}$ are depicted in Figure 2, $x_{i}$ is the coordinate of node $i$, while $W_{i}^{Q}(t)$ and $W_{i}^{M}(t)$ are defined as the "ENF functions" for the shear and moment at node $i$, with $Q$ and $M$ corresponding to shear and moment, respectively.

By establishing a finite element model of the bridge girder and converting the moving force into the ENFs on all the nodes, the moving force problem is converted to a classical time-history analysis problem with a random excitation history at each node. The construction of the traffic excitation SDM can then be based on the ENFs; for example, the equivalent forces at node $i$ are now

$$
\begin{gathered}
F_{i}^{Q}(t)=-\sum_{j=1}^{N(t)} P_{j} W_{i}^{Q}\left(t-\tau_{j}\right) \\
F_{i}^{M}(t)=-\sum_{j=1}^{N(t)} P_{j} W_{i}^{M}\left(t-\tau_{j}\right)
\end{gathered}
$$

Equations (5) and (6) indicate that $F_{i}^{Q}(t)$ and $F_{i}^{M}(t)$ are both filtered Poisson processes.

Expressing the ENFs as a vector,

$F(t)=$

$\left\{\begin{array}{llllllll}F_{1}^{Q}(t) & F_{2}^{Q}(t) & \cdots & F_{m}^{Q}(t) & F_{1}^{M}(t) & F_{2}^{M}(t) & \cdots & F_{m}^{M}(t)\end{array}\right\}^{\mathrm{T}}$

where $\mathrm{T}$ denotes the transpose and $m$ is the total number of nodes. Applying Campbell's theorem (Parzen, 1962) for filtered Poisson processes, and transforming to the frequency domain, the SDM of the excitation is obtained in partition matrix format as

$$
\mathbf{S}_{\mathbf{F F}}(\omega)=\left[\begin{array}{l|l}
\mathbf{S}_{\mathbf{Q Q}} & \mathbf{S}_{\mathbf{Q M}} \\
\hline \mathbf{S}_{\mathbf{M Q}} & \mathbf{S}_{\mathbf{M M}}
\end{array}\right]_{2 m \times 2 m}
$$

in which, for example, the $(i, j)$ term of the sub-matrix $\mathbf{S}_{\mathbf{Q Q}}(\omega)$ is

$$
\begin{aligned}
{\left[\mathbf{S}_{\mathbf{Q Q}}\right]_{i j}(\omega)=} & \lambda E\left(P^{2}\right) \int_{-\infty}^{\infty} \mathrm{e}^{-i \omega \tau} d \tau \\
& \times \int_{-\infty}^{\infty} W_{i}^{Q}(t) W_{j}^{Q}(t+\tau) d t
\end{aligned}
$$

where $E\left(P^{2}\right)$ is the mean square of random variable $P$.

\subsection{Limitation of the conventional excitation model}

Consider an example in which the nodes of the beam elements are equally spaced in the finite element model of a bridge. According to Equation (3), $W_{i}^{Q}(t)$ and $W_{j}^{Q}(t)$ have exactly the same shape (provided that $i$ or $j$ is not the end node of the bridge), and $W_{j}^{Q}(t)$ is the delayed version of $W_{i}^{Q}(t)$ with time lag $s=\left(x_{j}-x_{i}\right) / v$, that is, $W_{j}^{Q}(t)=W_{i}^{Q}(t-s)$. Therefore,

$$
\left[\mathbf{S}_{\mathbf{Q Q}}\right]_{i j}(\omega)=\mathrm{e}^{-i \omega s}\left[\mathbf{S}_{\mathbf{Q Q}}\right]_{i i}(\omega)
$$




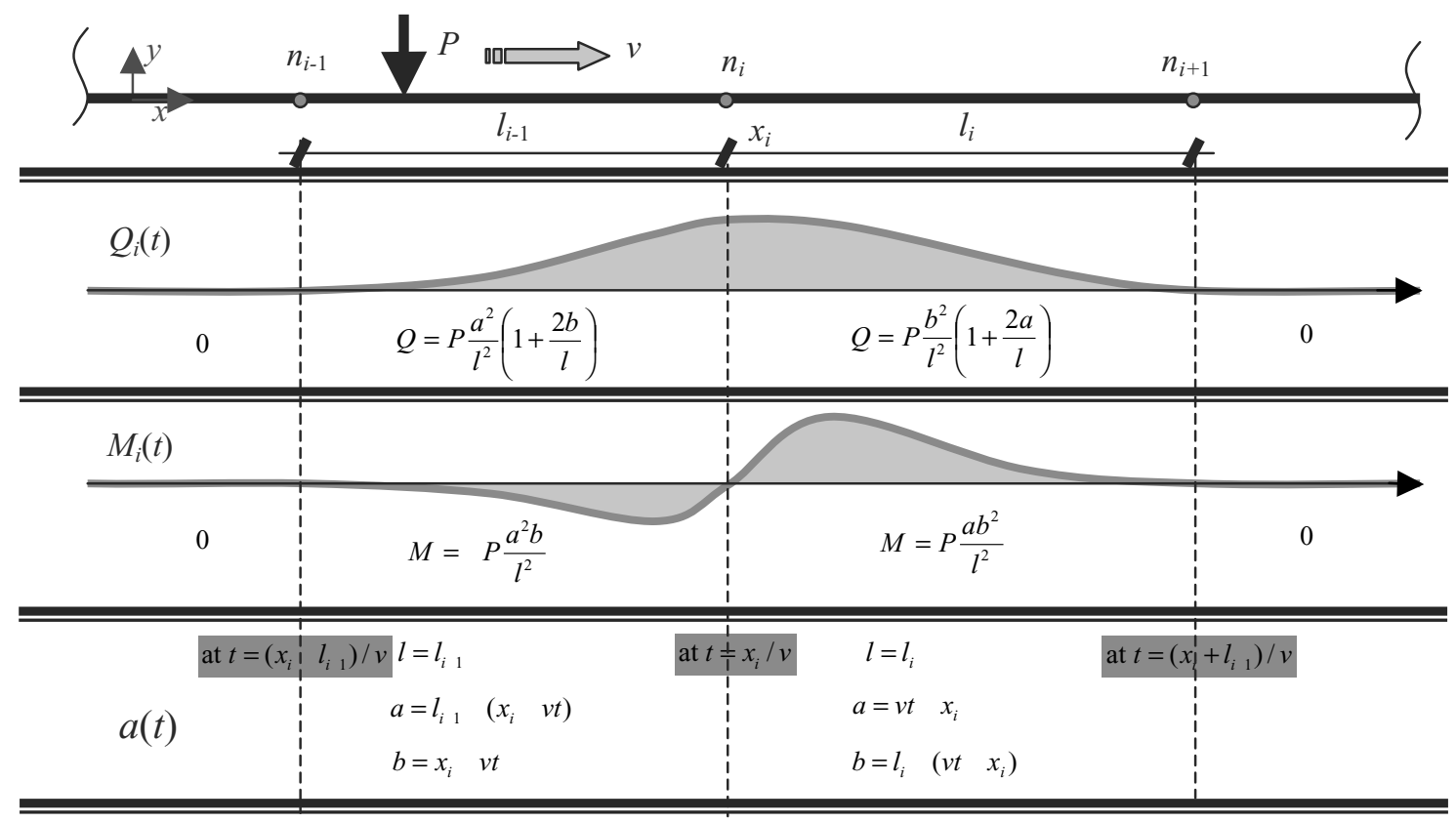

Fig. 2. Equivalent nodal forces (ENFs) function at node $i$.

Equation (10) shows two important characteristics of the excitation SDM: (1) the off-diagonal terms obtained from the moving load model have the same order of amplitudes as the diagonal terms and (2) the off-diagonal terms contain phase shifting information, which varies with $\omega$ and represents the spatial correlation between ENFs at different locations. Note that this nondiagonal excitation SDM is significantly different from the conventional excitation model, whose SDM is assumed constant and usually proportional to a unit matrix $\mathbf{I}$.

Many of the existing output-only system identification techniques are based on the conventional excitation model, among which the FDD technique is used as an example in this study to show its limitations. It will be shown numerically that the application of the FDD technique to structural responses due to spatially correlated excitations leads to misleading identification results of the structural dynamic properties (such as natural frequencies and mode shapes).

The spectrum of the response $\mathbf{S}_{\mathbf{Y Y}}(\omega)$ (Clough and Penzien, 1975) is

$$
\mathbf{S}_{\mathbf{Y Y}}(\omega)=\mathbf{H}(\omega) \mathbf{S}_{\mathbf{F F}}(\omega) \mathbf{H}^{*}(\omega)
$$

where $\mathbf{H}(\omega)$ is the transfer matrix of the structure and "*" denotes the complex conjugate transpose. The modal decomposition gives

$$
\mathbf{H}(\omega)=\boldsymbol{\Phi} \boldsymbol{\Lambda}(\omega) \boldsymbol{\Phi}^{\mathrm{T}}
$$

where $\boldsymbol{\Phi}$ is the mode shape matrix normalized so that it is unitary, and the diagonal matrix $\Lambda(\omega)$ contains the trans- fer function of each mode. When the excitation spectrum $\mathbf{S}_{\mathbf{F F}}(\omega)$ is proportional to a unit matrix $\mathbf{I}, \mathbf{S}_{\mathbf{F F}}(\omega)=c \mathbf{I}$, and

$$
\mathbf{S}_{\mathbf{Y Y}}(\omega)=c \boldsymbol{\Phi} \boldsymbol{\Lambda}(\omega) \boldsymbol{\Lambda}^{*}(\omega) \boldsymbol{\Phi}^{\mathrm{T}}
$$

Thus, the singular value decomposition (Marple, 1987) of $\mathbf{S}_{\mathbf{Y Y}}(\omega)$ leads to the structural modal properties of both $\Phi$ and $\Lambda(\omega)$. In the vicinity of the $i$ th mode, for example, $\left|\boldsymbol{\Lambda}\left(\omega_{i}\right)\right|^{2}$ will be the dominating singular value and the $i$ th mode shape $\phi_{i}$ will be the first singular vector obtained by the decomposition.

In Brincker et al. (2001), this FDD technique is extended to cases where $\mathbf{S}_{\mathbf{F F}}(\omega)$ is not proportional to a unit matrix I. However, two conditions are required for this technique to work: (1) the structure should have lightly-damped distinct modes, and (2) near the natural frequency of each mode, the input spectrum $\mathbf{S}_{\mathbf{F F}}(\omega)$ should be almost constant. Indeed, one key assumption of output-only techniques is that the input SDM is essentially constant within the frequency range of interest so that there is neither amplitude variation nor phase shifting due to the excitation to overlay the structural frequency response. Equation (10) has shown that the spatial correlation of the excitation results in a nondiagonal, frequency-dependent $\mathbf{S}_{\mathbf{F F}}(\omega)$, violating the white noise assumption of the FDD technique.

The above discussion will be illustrated by a numerical example. A finite element model has been developed based on an in-service three-span prestressed box-girder concrete highway bridge, the Jamboree Road 


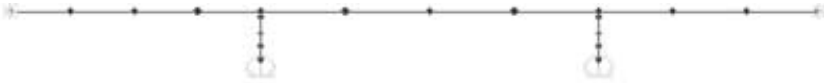

(a) Finite element model of a bridge

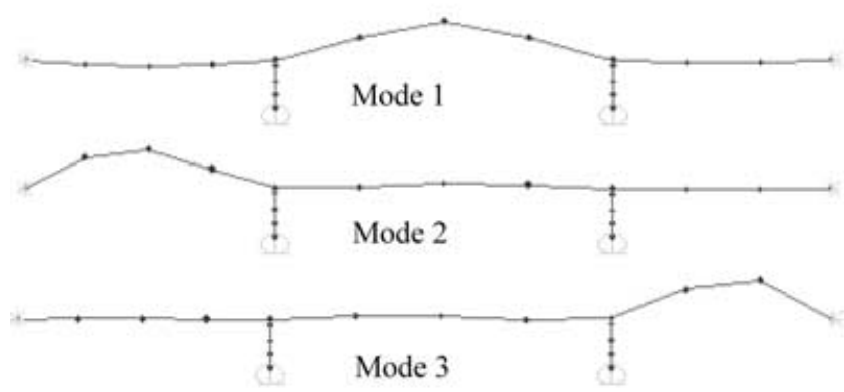

(b) The first three mode shapes

Fig. 3. Finite element model and vibration modes of a bridge.

Overcrossing as shown in Figure 3a (Feng et al., 2004). The girder and the columns are modeled by frame elements, with each node having two degrees of freedom, namely, the transition and the in-plane rotation. Eigen analysis is performed in SAP2000 (CSI, 1996) to obtain the natural frequencies and mode shapes; the first three modes are illustrated in Figure 3b. Damping ratios for the three modes are assigned $\zeta_{1}=2 \%, \zeta_{2}=4 \%$, and $\zeta_{3}=5 \%$, respectively, to obtain $\Lambda(\omega)$. By the singular value decomposition of Equation (13) (with $c=1$ ) at each frequency $\omega$, the true singular value, as a function of $\omega$, is the dominating diagonal element of $\Lambda(\omega) \Lambda^{*}(\omega)$, shown by the solid line in Figure 4. The true mode shape $\phi(\omega)$, associated with the dominating mode at frequency $\omega$, is also obtained from the first singular vector.
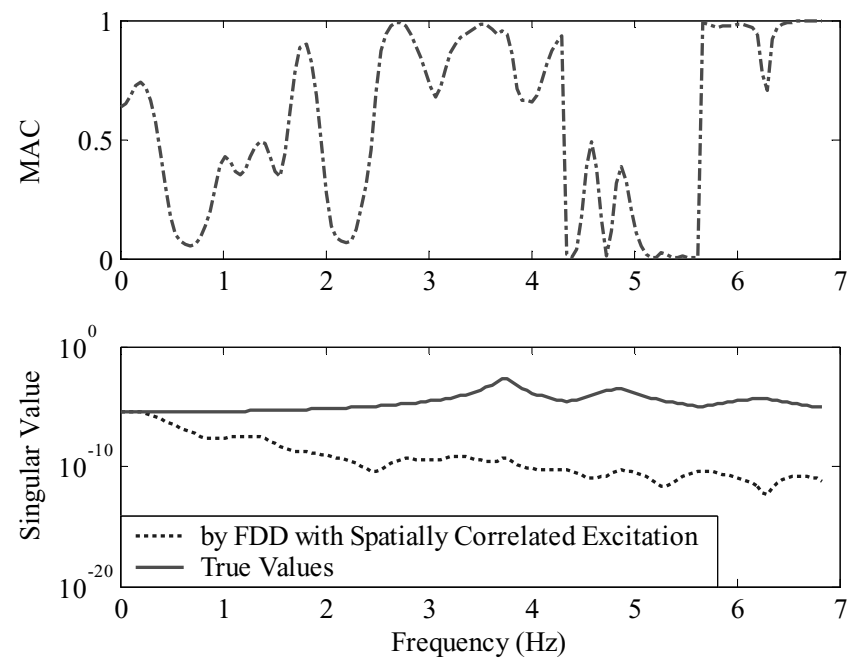

Fig. 4. The FDD results with the physics-based excitation SDM.
It is straightforward to construct the physics-based spatially correlated excitation SDM following the procedure outlined in Section 2.2. For this finite element model, the excitation SDM due to moving vehicles (assuming constant tire force) with a speed $17.8 \mathrm{~m} / \mathrm{s}$, which is the normal vehicle speed for this bridge, is calculated with constants $\lambda$ and $E\left(P^{2}\right)$ set to 1 . By inserting the constructed $\mathbf{S}_{\mathbf{F F}}$ into Equation (11) and performing the singular value decomposition of $\mathbf{S}_{\mathbf{Y Y}}(\omega)$, the singular values and singular vectors are obtained from the FDD technique and compared to the true structural properties in Figure 4. The MAC value (Ewins, 1985), an index of the similarity between two shapes, is calculated at each point of $\omega$ between the resulting singular vector and the true mode shape,

$$
\operatorname{MAC}(i, j)=\frac{\left|\sum_{k=1}^{n}\left(\varphi_{j}\right)_{k}\left(\varphi_{i}\right)_{k}^{*}\right|^{2}}{\left(\sum_{k=1}^{n}\left(\varphi_{j}\right)_{k}\left(\varphi_{j}\right)_{k}^{*}\right)\left(\sum_{k=1}^{n}\left(\varphi_{i}\right)_{k}\left(\varphi_{i}\right)_{k}^{*}\right)}
$$

Figure 4 confirms the results in the literature that the moving force with a normal speed can only excite the low frequency vibration of the bridge (Chen and Duan, 1999). More importantly, it is found that the peaks of the singular value (on the dotted line) no longer indicate the natural frequencies of the bridge. Some true peaks are suppressed (e.g., the peak at $6.24 \mathrm{~Hz}$ ); some pseudo peaks bear no connection to the mode resonances. It can also be observed that the singular vector at mode 2 $(4.86 \mathrm{~Hz})$ presents low similarity to the true mode shapes $(\mathrm{MAC} \ll 1)$. These results suggest that directly applying the FDD technique on the vibration responses excited by spatially correlated excitation might result in falsely identified structural dynamic properties.

\section{FEASIBILITY OF SYSTEM IDENTIFICATION USING THE PHYSICS-BASED EXCITATION MODEL}

For the purpose of long-term monitoring of bridge structural performance, structural properties are often identified from measured dynamic responses of the bridge subjected to excitations from moving vehicles. These excitations are difficult, if not impossible, to measure, and are spatially correlated. As available system identification techniques assume excitations to be spatially uncorrelated white noise, it is of theoretical interest and practical importance to develop a system identification technique for systems subjected to unknown but spatially correlated excitations. This article discusses the feasibility of such an identification technique currently being developed by the authors. 


\subsection{Parameterization of the physics-based traffic excitation model}

As stated earlier, the excitation process at node $i$ is the time-delay of the process at a preceding node $j$, that is, $F_{i}(t)=F_{j}\left(t-\Delta t_{i j}\right)$, where $\Delta t_{i j}=\left(x_{i}-x_{j}\right) / v$. Expressing in a discrete time format, the excitation load vector becomes

$$
\begin{aligned}
\mathbf{F}(n)= & \left\{\begin{array}{c}
F_{1}(n) \\
F_{2}(n) \\
\vdots \\
F_{m}(n)
\end{array}\right\}=\left\{\begin{array}{c}
1 \\
0 \\
\vdots \\
0
\end{array}\right\} F(n)+\left\{\begin{array}{c}
0 \\
1 \\
\vdots \\
0
\end{array}\right\} \\
& \times F\left(n-s_{21}\right)+\cdots+\left\{\begin{array}{c}
0 \\
0 \\
\vdots \\
1
\end{array}\right\} F\left(n-s_{m 1}\right)
\end{aligned}
$$

where $F(n)=F_{1}(n)$; the lag $s_{i j}$ of channel $i$ with respect to channel $j$ is defined such that $s_{i j} \cdot \Delta T=\left(l_{i}-l_{j}\right) / v$, where $\Delta T$ is the sampling interval.

Introducing a backward operator $B$ such that $B \cdot F(n)=F(n-1)$, Equation (15) can be rewritten as

$$
\begin{aligned}
\mathbf{F}(n) & =\left\{\begin{array}{c}
F_{1}(n) \\
F_{2}(n) \\
\vdots \\
F_{m}(n)
\end{array}\right\}=\left\{\begin{array}{c}
1 \\
B^{s_{21}} \\
\vdots \\
B^{s_{m 1}}
\end{array}\right\} F(n) \\
& =\left[\begin{array}{ccc}
1 & \\
B^{s_{21}} & 1 & \\
\vdots & & \ddots \\
B^{s_{m 1}} & & 1
\end{array}\right]\left\{\begin{array}{c}
F(n) \\
0 \\
\vdots \\
0
\end{array}\right\}
\end{aligned}
$$

In this parameterization, the spatial correlation between excitation processes at different locations is represented as the interchannel delay, which in turn depends on the vehicle speed and the distance between the nodes.

\subsection{Identifiability of the multichannel ARMA model}

An essential concern for a system identification technique is whether it can lead to a unique solution to the identification problem, which is referred to as the identifiability (Priestley, 1981). The structural dynamic responses depend not only on the structural properties but also on the characteristics of the excitations. Therefore, given a response time history, there exist infinite combinations of structural properties and excitations that can produce the same response. To uniquely identify the structural properties (or the excitation characteristics), knowledge on the excitations (or the structural properties) should be provided, or to identify the structural properties and the excitation characteristics simultaneously from the responses, partial knowledge on both should be available a priori. The a priori knowledge should be adequate to confine the identification to a unique solution.

In general, $F(n)$ in Equation (16) is colored noise. But when the vehicle speed is not very low, $F(n)$ tends to be an impulse-like excitation. At this stage of research, it is treated as a white noise disturbance. Representing the structural system by second order difference equations, the dynamics of the bridge subjected to traffic excitations can be formulated in a multichannel ARMA model as

$$
\begin{aligned}
\mathbf{Y}(n) & +\mathbf{a}_{1} \mathbf{Y}(n-1)+\mathbf{a}_{2} \mathbf{Y}(n-2) \\
= & \mathbf{A F}(n)=\varepsilon(n)+\mathbf{A} \mathbf{b}_{s_{21}} \mathbf{A}^{-1} \varepsilon\left(n-s_{21}\right) \\
& +\cdots+\mathbf{A b}_{s_{m 1}} \mathbf{A}^{-1} \varepsilon\left(n-s_{m 1}\right)
\end{aligned}
$$

or

$$
\boldsymbol{\alpha}(B) \mathbf{Y}(n)=\boldsymbol{\beta}(B) \varepsilon(n)
$$

where $\mathbf{Y}(n)$ is the vector of structural response; $\mathbf{a}_{1}, \mathbf{a}_{2}$, and $\mathbf{A}$ are system matrices related to the structural properties such as the mass, stiffness, and damping matrices, $\mathbf{A}$ is the normalization matrix such that the coefficient of $\mathbf{Y}(n)$ is a unit matrix $\mathbf{I} ; \varepsilon(n)$ is the vector of white noise disturbance, $\varepsilon(n)=\mathbf{A} \cdot\{F(n), 0, \ldots, 0\}^{\mathrm{T}}$ with zero mean and the variance matrix

$$
E\left[\varepsilon(n) \varepsilon^{\mathrm{T}}(n)\right]=\mathbf{A}\left[\begin{array}{cccc}
\sigma^{2} & 0 & \cdots & 0 \\
0 & 0 & & \\
\vdots & & \ddots & \vdots \\
0 & & \cdots & 0
\end{array}\right] \mathbf{A}^{\mathrm{T}}
$$

$\mathbf{b}_{s_{i 1}}$ is the matrix that has only one nonzero entry $\mathbf{b}_{s_{i 1}}(i, 1)=1 ; \boldsymbol{\alpha}(B)=\mathbf{I}+\mathbf{a}_{1} B+\mathbf{a}_{2} B^{2}$, and $\boldsymbol{\beta}(B)=$ $\mathbf{A}\left(\mathbf{I}+\mathbf{b}_{s_{21}} B^{s_{21}}+\cdots+\mathbf{b}_{s_{m 1}} B^{s_{m 1}}\right) \mathbf{A}^{-1}$.

The identifiability of multichannel ARMA models has been studied by many authors (Hannan, 1969, 1976; Grewal and Glover, 1976; Söderström et al., 1976; Swami et al., 1994), with classical conclusions summarized in Priestley (1981). As the structures are stable, zeros of $|\boldsymbol{\alpha}(B)|$ lie outside of the unit circle; moreover, $|\boldsymbol{\beta}(B)|$ $=1$ (by direct calculation of the determinant), the MA part is thus always invertible; $\boldsymbol{\alpha}(B)$ and $\boldsymbol{\beta}(B)$ have no common left devisors except constants (no common zeros). As a result, this multichannel ARMA model is identifiable.

In summary, by parameterizing the spatial correlation of the excitation as the interchannel delay in the MA part, the structural responses conform to a parameterized multichannel ARMA model, which is identifiable. It is thus possible to uniquely estimate the structural properties based on this parameterization. 


\subsection{Gray-box system identification}

The structural properties and the excitation characteristics are not totally unknown, or a "black box" to us. By parameterization of the MA part, our knowledge about the nature of the traffic excitation, that is, its spatial correlation, is embedded in the model. Similarly, our knowledge about the structural properties can be built into the model by parameterizing the AR part. For example, for a lumped-mass system, the mass matrix should be diagonal; for Rayleigh damping, the damping matrix is a linear combination of the mass and the stiffness matrices; for some columns or beams, they are of the same design configuration and when this information goes into the stiffness matrix, its entries will be related in a prescribed way. Such parameterizations lead to a "gray-box" identification technique (Ljung, 1987). When compared to the black-box identification, gray-box techniques reduce the number of the unknowns, enhance the identifiability, and usually lead to more reliable results.

A numerical example is given to demonstrate the feasibility of the proposed gray-box identification technique with the proposed physics-based excitation. In this example, as a proof of concept, a simple bridge structure is adopted. The spatial correlation of the excitation is deliberately introduced by applying the same white noise excitation history at different locations (nodes 1-7) on the deck, but with different delays. This is equivalent (in frequency domain) to physically hammering those nodes sequentially during the test. It is noted that the identification procedure and the excitation model are applicable to general bridge structures.

The example one-span bridge is supported on two abutments represented by vertical and rotational springs, see Figure 5. It has a total length of $30 \mathrm{~m}$ and is equally divided into six elements. The baseline properties of this bridge are elastic modulus $\bar{E}=2.5 \times 10^{10} \mathrm{~Pa}$, mass density $d=2,400 \mathrm{~kg} / \mathrm{m}^{3}$, cross-sectional area $A=6.0 \mathrm{~m}^{2}$, and moment of inertia $\bar{I}=0.5 \mathrm{~m}^{4}$; both abutments have the same stiffness, $2.0 \times 10^{11} \mathrm{~N} / \mathrm{m}$ for the vertical springs and $2.0 \times 10^{10} \mathrm{~N}-\mathrm{m} / \mathrm{rad}$ for the rotational springs. Analytical modes are obtained, with the natural frequencies of the first three modes as $3.42,9.48$, and $18.74 \mathrm{~Hz}$, respectively, and their corresponding mode shapes plotted in Figure 5. Rayleigh type damping is assumed in the response simulation, as well as in the identification procedure of the structural properties, with the damping ratios of the first two modes being $1 \%$ and $2 \%$, respectively.

Three cases are considered and the dynamic responses of the bridge are simulated. In Case 1, the structural properties are assigned the same values as those in the baseline, and the delay between excitations at two adjacent nodes is 0.01 seconds (associated with a vehicle speed of $50 \mathrm{~m} / \mathrm{s}$ ); while in Case 2, the delay between two adjacent nodes is 0.40 seconds (associated with a speed of $12.5 \mathrm{~m} / \mathrm{s}$ ), with the baseline structural properties. In Case 3, the structural properties remain the same except that $\bar{I}=0.6 \mathrm{~m}^{4}$, and the excitation delay is still $0.40 \mathrm{sec}-$ onds between two adjacent nodes. The simulation was done in the time domain to obtain the time histories of the vertical acceleration responses at all the nodes.

In Figure 6, the Fourier spectra of the acceleration responses at node 4 in Cases 1 and 2 are plotted for comparison. In Case 1, because the delay between two adjacent nodes is short ( 0.01 seconds) the excitations at all nodes are almost in phase. One can see on the dotted line two distinct peaks at about 3.4 and $18.7 \mathrm{~Hz}$, corresponding to the first and third modes, while the second mode around $9.5 \mathrm{~Hz}$ is suppressed, as expected, due to the symmetry of this structure. It is more interesting to note that in Case 2, in which the excitation delay is longer (0.40 second) and its effect is more observable, the first mode is also suppressed (around $3.4 \mathrm{~Hz}$ ), while the peak of the third mode is split into two pseudo peaks, even though the structure remains unchanged. This simulation demonstrates the effect of the interchannel delay of the excitation.

Now given the simulated responses in Case 3 (among which, spectra of responses at nodes 2,3 , and 4 are plotted in the dotted line in Figure 7), proceed to identify the structural properties, as well as the interchannel delay of the excitation. As a proof of this concept, the

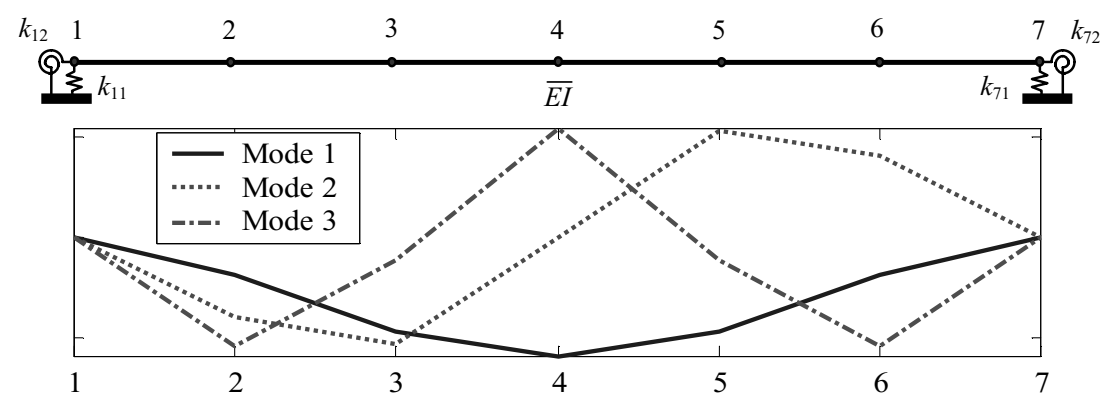

Fig. 5. Example bridge and its first three mode shapes. 


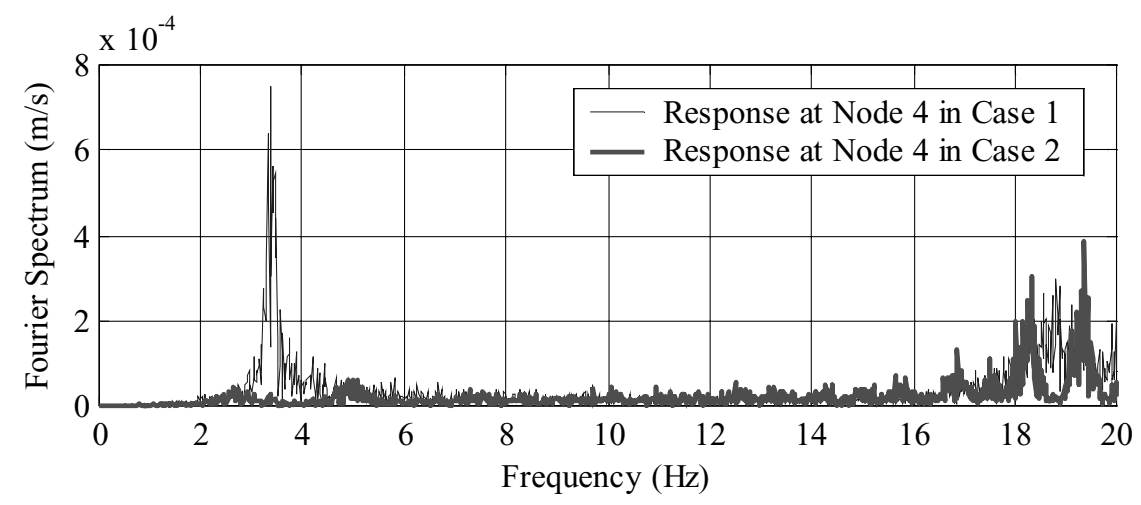

Fig. 6. Comparison of response spectra for Case 1 and Case 2.

cross-sectional moment of inertia $\bar{I}$ is the only unknown structural property to be identified in addition to the unknown interchannel delay. As damping is of Rayleigh type, the damping matrix, though unknown, is a function of $\bar{I}$. The damping ratios of the first two modes are assumed to be a priori known, being 1\% and 2\% respectively, while the Rayleigh coefficients are being updated in the identification procedure to maintain these modal dampings.

The identification algorithm is outlined as follows: first, for a preset interchannel delay $s$, search for a combination of structural properties that minimizes a loss function. Then, change the delay $s$ to cover a grid of possible values. For each of the delays, carry out the structural property searching as in the first step. Finally, from all the possible delays (and the structural property combinations associated with them), find the one that has the minimum loss function; this is the identification result.

If a tentative combination of structural properties (a tentative $\bar{I}$ in this example) is assumed, together with the preset delay, all parameters in the ARMA model are available so that a forward analysis can be performed to obtain its response spectra. The loss function $f(I, s)$ is defined as an inner product of the error matrix between the given response spectra and those from the forward analysis of the ARMA model:

$$
f(I, s)=\log \left(\operatorname{tr}\left\{\left[\mathbf{S}_{g}-\mathbf{S}_{t}(I, s)\right]^{*}\left[\mathbf{S}_{g}-\mathbf{S}_{t}(I, s)\right]\right\}\right)
$$

where $\mathbf{S}_{g}$ is the matrix of the given response spectra, with its $(i, j)$ element being the Fourier spectrum of the response at $j$ th node at frequency $\omega_{i}$; whereas $\mathbf{S}_{t}$ is the matrix of response spectra, whose elements are arranged in the same way as those in $\mathbf{S}_{g}$, from the ARMA model with the tentative parameters. The function $\operatorname{tr}()$ is the trace of a matrix. The minimization of the loss function with respect to $\bar{I}$ follows a sequential quadratic programming (SQP) procedure, a standard subroutine in the optimization toolbox of MatLab (Hock and Schittkowski,
1983). During each minimization procedure for a delay $s$, the initial value of $\bar{I}$ is set to $0.5 \mathrm{~m}^{4}$. This algorithm is programmed in MatLab, yielding the results shown in Table 1.

The identified results are $s=0.40$ seconds and $\bar{I}=$ $0.5926 \mathrm{~m}^{4}$. They match their target values well. The theoretical response spectra of the ARMA model augmented with these identified results are plotted as a solid
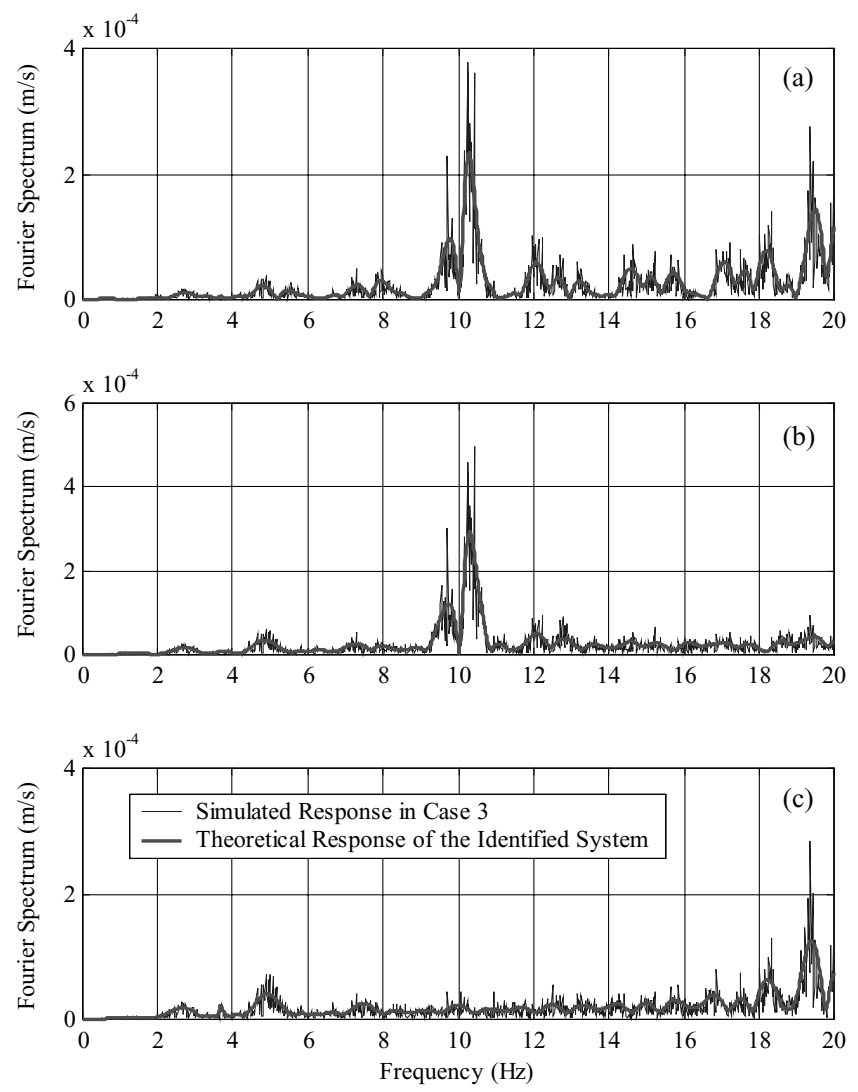

Fig. 7. Response spectra in Case 3 and the identified system (a) at node 2 , (b) at node 3 , and (c) at node 4 . 
Table 1

Identification results using the gray-box technique

\begin{tabular}{lcc}
\hline $\begin{array}{l}\text { Preset } \\
\text { delay } \\
\text { (seconds) }\end{array}$ & $\begin{array}{c}\text { Structural } \\
\text { properties } \bar{I}\left(m^{4}\right) \\
\text { (resulted) }\end{array}$ & $\begin{array}{c}\text { Value } \\
\text { of loss } \\
\text { function }\end{array}$ \\
\hline 0.20 & 1.471 & -90.68 \\
0.30 & 0.5903 & -93.00 \\
0.40 & 0.5926 & -97.70 \\
0.50 & 0.5769 & -91.75 \\
0.60 & 0.9390 & -90.78 \\
\hline
\end{tabular}

line in Figure 7, where one can observe that the identified system produces very close response spectra to the given data. This example demonstrates the feasibility of the gray-box technique to identify structural properties and excitation characteristics when the excitation is not measured or completely known but is spatially correlated.

\section{CONCLUSION}

In this article, traffic excitation is physically modeled as a stationary stream of vehicles, arriving in accordance with a Poisson process, traversing an elastic beam. The trafficinduced excitation on a bridge is found to be spatially correlated because its process at one location is of the same profile as the processes at preceding locations but with a time-delay. This spatial correlation results in an excitation SDM with nonzero off-diagonal terms varying with frequency. This finding is in contrast to the conventional approach of assuming a spatially uncorrelated white noise excitation model for system identification when only the structural response is available. Numerical results on the system identification of a bridge structure show that direct application of the FDD technique, an identification technique based on the conventional excitation model, to responses due to spatially correlated excitations leads to misleading conclusions. A physicsbased model for traffic excitation on highway bridges, taking into account the spatial correlation, is thus proposed in this article.

Based on this physics-based excitation model, an output-only gray-box system identification technique is further proposed and its feasibility is demonstrated. In this technique, the structural responses conform to an ARMA model, whose parameterized MA part is the physics-based excitation model with our a priori knowledge about its spatial correlation embedded. The identifiability of the ARMA model so formulated is assured so that a unique identification of the structural proper- ties and the excitation characteristics can be achieved. A numerical example of a bridge structure subjected to a spatially correlated excitation is presented to demonstrate the feasibility of the proposed identification technique. With the proposed physics-based excitation model and the gray-box identification technique, information collected about the traffic such as vehicle arriving times and speeds will directly contribute to improving the structural property identification.

\section{REFERENCES}

Asmussen, J. C. \& Brincker, R. (1996), Estimation of frequency response function by random decrement, in Proceedings of the 14th IMAC, Dearborn Michigan, 246-52.

Brincker, R., Zhang, L. \& Andersen, P. (2001), Model identification of output-only systems using frequency domain decomposition, Smart Materials and Structures, 10, 441-5.

Caicedo, J. M., Dyke, S. J. \& Johnson, E. A. (2004), Natural excitation technique and eigensystem realization algorithm for phase I of the IASC-ASCE benchmark problem: Simulated data, ASCE Journal of Engineering Mechanics, 130(1), 49-60.

Cebon, D. (1999), Handbook of Vehicle-Road Interaction, Swets \& Zeitlinger Publishers, Lisse, The Netherlands.

Chen, W.-F. \& Duan, L. eds. (1999), Bridge Engineering Handbook, CRC Press, Boca Raton.

Clough, R. W. \& Penzien, J. (1975), Dynamics of Structures, McGraw-Hill, New York.

CSI (1996), SAP2000 Analysis Reference, Computers and Structures Inc., Berkeley, California.

Ewins, D. J. (1985), Modal Testing: Theory and Practice, Wiley, New York.

Feng, M. Q. \& Kim, J. M. (1998), Identification of a dynamic system using ambient vibration measurements, ASME Journal of Applied Mechanics, 65(2), 1010-23.

Feng, M. Q., Kim, D. K., Yi, J.-H. \& Chen, Y. (2004), Baseline models for bridge performance monitoring, ASCE Journal of Engineering Mechanics, 130(5), 562-69.

Garibaldi, L., Giorcelli, E. \& Piombo, B. A. D. (1998), ARMAV techniques for traffic excited bridges, ASME Journal of Vibration and Acoustics, 120(3), 713-18.

Grewal, M. S. \& Glover, K. (1976), Identifiability of linear and nonlinear dynamical systems, IEEE Transactions on Automatic Control, 833-37.

Hannan, E. J. (1969), The identification of vector mixed autoregressive-moving average systems, Biometrika, 56(1), 223-25.

Hannan, E. J. (1976), The identification and parameterization of ARMAX and state space forms, Econometrica, 44(4), 713-23.

Hibbeler, R. C. (2002), Structural Analysis, Prentice-Hall Inc., Upper Saddle River, New Jersey.

Hock, W. \& Schittkowski, K. (1983), A comparative performance evaluation of 27 nonlinear programming codes, Computing, 30, 335.

Huang, C. S. (2001), Structural identification from ambient vibration measurement using the multivariate AR model, Journal of Sound and Vibration, 241(3), 337-59.

James, G. H., III, Garne, T. G. \& Mayes, R. L. (1996), Modal parameter extraction from large operating structures using 
ambient excitation, in Proceedings of the 14th IMAC, Dearborn Michigan, pp. 77-83.

Jenson, J. L., Kirkegaard, P. H. \& Brincker, R. (1992), Modal and wave load identification by ARMA calibration, $A S C E$ Journal of Engineering Mechanics, 118(6), 1268-73.

Ljung, L. (1987), System Identification: Theory for the User, Prentice-Hall, Inc., Englewood Cliffs, New Jersey.

Marple, S. L., Jr. (1987), Digital Spectral Analysis, PrenticeHall Inc., Englewood Cliffs, New Jersey.

Pan, T.-C. \& Li, J. (2002), Dynamic vehicle element method for transient response of coupled vehicle-structure systems, ASCE Journal of Structural Engineering, 128(2), 21423.

Parzen, E. (1962), Stochastic Processes, Holden-Day Inc., San Francisco, California.

Peeters, B., Maeck, J. \& De Roeck, G. (2001), Vibration-based damage detection in civil engineering: Excitation sources and temperature effects, Smart Materials and Structures, 10, 518-27.

Pesterev, A. V., Bergman, L. A., \& Tan, C. A. (2004), A novel approach to the calculation of pothole-induced contact forces in MDOF vehicle models, Journal of Sound and Vibration, 275, 127-49.

Pesterev, A. V., Yang, B., Tan, C. A., \& Bergman, L. A. (2003) Assessment of pothole-induced tire forces in a general linear vehicle model, in Proceedings of the ASCE Engineering Mechanics Conference, Seattle, Washington.
Priestley, M. B. (1981), Spectral Analysis and Time Series, vol. 2: Multivariate Series, Prediction and Control, Academic Press, London.

Schenk, C. A. \& Bergman, L. A. (2003), Response of continuous system with stochastically varying surface roughness to moving load, ASCE Journal of Engineering Mechanics, 129(7), 759-68.

Shen, F., Zheng, M., Feng, D. \& Xu, F. (2003), Using the cross-correlation technique to extract modal parameters on response-only data, Journal of Sound and Vibration, 259(5), 1163-79.

Shinozuka, M. \& Kobori, T. (1972), Fatigue analysis of highway bridges, in Proceedings of the Journal of Structural and Civil Engineering, 208, 137-48.

Söderström, T., Ljung, L. \& Gustavsson, I. (1976), Identifiability conditions for linear multivariable system operating under feedback, IEEE Transactions on Automatic Control, 21(6), 837-40.

Swami, A., Giannakis, G. \& Shamsunder, S. (1994), Multichannel ARMA processes, IEEE Transactions on Signal Processing, 42(4), 898-912.

Turner, J. D. \& Pretlove, A. J. (1988), A study of the spectrum of traffic-induced bridge vibration, Journal of Sound and Vibration, 122(1), 31-42.

Yang, B., Tan, C. A. \& Bergman, L. A. (2000), Direct numerical procedure for solution of moving oscillator problems, ASCE Journal of Engineering Mechanics, 126(5), 462-69. 